Revue internationale P.M.E.

Économie et gestion de la petite et moyenne entreprise

\title{
Approche cognitive de la gestion stratégique dans une entreprise monodécideur : le cas de l'entrepreneur agricole
}

\section{Sophie Allain}

Volume 12, numéro 1-2, 1999

URI : https://id.erudit.org/iderudit/1008649ar

DOI : https://doi.org/10.7202/1008649ar

Aller au sommaire du numéro

Éditeur(s)

Presses de l’Université du Québec

ISSN

0776-5436 (imprimé)

1918-9699 (numérique)

Découvrir la revue

Citer cet article

Allain, S. (1999). Approche cognitive de la gestion stratégique dans une entreprise monodécideur : le cas de l'entrepreneur agricole. Revue internationale P.M.E., 12(1-2), 31-60. https://doi.org/10.7202/1008649ar

\section{Résumé de l'article}

Dans le domaine de la gestion stratégique des PME, une des questions clés est de savoir comment s'établit le lien entre les idées contenues dans la vision stratégique du dirigeant et les décisions réelles qui sont prises. On aborde ici cette question par une approche cognitive ; il s'agit de comprendre pourquoi et comment un individu prend ses décisions. Et on considère que ce n'est qu'en revenant à la manière même dont celui-ci interprète sa situation qu'on peut y parvenir. On choisit le cas de l'entreprise agricole pour prospecter cette voie, car il s'agit d'une très petite entreprise, généralement pilotée par un seul décideur; vision stratégique et décisions concrètes relèvent donc de la même personne. On propose tout d'abord le schéma suivant des décisions :

l'agriculteur a des représentations stables, qui sont, d'une part, une vision stratégique de son entreprise lui fournissant les éléments de sa politique générale et, d'autre part, des programmes d'action lui permettant de prendre des décisions opérationnelles à caractère répétitif, donc, d'assurer le fonctionnement courant de son entreprise; le couplage entre ces deux plans est assuré par les décisions de gestion stratégique qui sont, pour nous, les décisions qui gèrent le changement dans l'entreprise. On construit alors une grille d'analyse de ces décisions. Celles-ci sont vues comme suivant à la fois une logique de résolution de problème et une logique d'adéquation, l'individu recherchant une solution satisfaisante, non connue a priori, en s'appuyant sur les représentations qui guident son action ; ces deux logiques obéissent à des principes de simplification, de continuité et de connexion étroite aux actions à mener au même moment. On applique cette grille au cas d'un agriculteur d'une région de grande culture française et on envisage pour finir quelques perspectives en matière d'aide à la décision et de recherche.
Ce document est protégé par la loi sur le droit d'auteur. L'utilisation des services d’Érudit (y compris la reproduction) est assujettie à sa politique d'utilisation que vous pouvez consulter en ligne.

https://apropos.erudit.org/fr/usagers/politique-dutilisation/ 


\title{
Approche cognitive de la gestion stratégique dans une entreprise monodécideur: le cas de l'entrepreneur agricole
}

Sophie ALLAIN

Institut national de la recherche agronomique

\author{
MOTS CLÉS
}

\section{Approche cognitive - Décision - Entrepreneur agricole \\ Gestion stratégique - Interprétation - PME - Rationalité limitée Représentation - Résolution de problème}

\begin{abstract}
RÉSUMÉ
Dans le domaine de la gestion stratégique des PME, une des questions clés est de savoir comment s'établit le lien entre les idées contenues dans la vision stratégique du dirigeant et les décisions réelles qui sont prises. On aborde ici cette question par une approche cognitive : il s'agit de comprendre pourquoi et comment un individu prend ses décisions. Et on considère que ce n'est qu'en revenant à la manière même dont celui-ci interprète sa situation qu'on peut y parvenir. On choisit le cas de l'entreprise agricole pour prospecter cette voie, car il s'agit d'une très petite entreprise, généralement pilotée par un seul décideur; vision stratégique et décisions concrètes relèvent donc de la même personne. On propose tout d'abord le schéma suivant des décisions : l'agriculteur a des représentations stables, qui sont, d'une part, une vision stratégique de son entreprise lui fournissant les éléments de sa politique générale et, d'autre part, des programmes d'action lui permettant de prendre des décisions opérationnelles à caractère répétitif, donc, d'assurer le
\end{abstract}

\section{L'AUTEURE}

Sophie Allain est ingénieure agronome et détentrice d'un doctorat en gestion de l'Institut national agronomique Paris-Grignon (INA-PG). Elle est également ingénieure de recherche à l'Institut national de la recherche agronomique en France et s'intéresse à l'analyse des processus de décision dans les organisations et, en particulier, à la gestion stratégique de l'entreprise agricole. Adresse: INRA- DADP, 147, rue de l'Université, 75338 Paris Cedex 07, France. 
fonctionnement courant de son entreprise; le couplage entre ces deux plans est assuré par les décisions de gestion stratégique qui sont, pour nous, les décisions qui gèrent le changement dans l'entreprise. On construit alors une grille d'analyse de ces décisions. Celles-ci sont vues comme suivant à la fois une logique de résolution de problème et une logique d'adéquation, l'individu recherchant une solution satisfaisante, non connue a priori, en s'appuyant sur les représentations qui guident son action; ces deux logiques obéissent à des principes de simplification, de continuité et de connexion étroite aux actions à mener au même moment. On applique cette grille au cas d'un agriculteur d'une région de grande culture française et on envisage pour finir quelques perspectives en matière d'aide à la décision et de recherche.

\section{ABSTRACT}

In the area of strategic management of small and medium-sized business, a keypoint is to know how ideas embedded in the strategic vision of manager are linked with decisions made. We treat this question through a behavioral approach: the purpose is to understand why and how somebody makes his decisions, and we consider that we can only achieve it by understanding how an actor interpretes his own situation. We choose the case of the farm to prospect this way, because it is a very small firm, generally leaded by a single manager: consequently, strategic vision and concrete decisions depend on the same person. We first propose the following pattern of decisions: the farmer has got stable representations, which are on the one hand a strategic vision of his firm, giving him elements of general policy, and on the other hand programmes of action for making operational, repetitive decisions, that is, to ensure the smooth ordinary running of the firm; the coupling between these two levels is made by strategic management decisions, which are decisions managing change in a firm. We then build a framework for the analysis of this kind of decisions : we consider that they follow both a logic of problem solving and a logic of appropriateness; the actor looks for a satisfying solution, unknown before, by leaning on representations which lead his action; both logics comply with the principles of simplification, continuity and link to the actions to lead at the same time. We apply this framework to the case-study of a farmer located in a French cropping region and we examine at the end some prospects for decision aid and research.

\section{RESUMEN}

En el campo de la gestión de estrategia de las PyMEs, una de las preguntas clave es de saber cómo se unen las ideas contenidas en la visión estratégica del dirigente y las decisiones tomadas. Abordamos aqui este asunto con un enfoque cognoscitivo: se trata de entender porque y cómo un individuo toma sus decisiones, y consideramos que sólo se puede comprender al estudiar la forma con la cual esta persona interpreta su situación. Elegimos la situación de la empresa agrícola con el fin de explorar esta vía, porque se trata de una empresa muy pequeña, generalmente dirigida por un solo jefe: visión estratégica y decisiones concretas pertenecen pues a la misma persona. Proponemos en primer lugar el esquema siguiente de las decisiones : el agricultor tiene unas representaciones estables, que son por una parte una visión estratégica de su empresa que le trae los elementos de su politica general, y por otra parte unos programas de acción permittiendóle 
tomar decisiones operativas, de caracter repetitivo, luego asegurar el funcionamiento corriente de su empresa; el acoplamiento entre estos dos planes está asegurado por las decisiones de gestión de estrategia que constituyen para nosotros las decisiones que administran el cambio en la empresa. Elaboramos entonces una red de análisis de estas decisiones : éstas están consideradas como siguiendo a la vez una lógica de resolución de un problema y una lógica de adecuación, el individuo investigando una solución satisfactoria, no conocida de ante mano, basandóse en las representaciones que guían su acción; estas dos lógicas obedecen a unas normas de simplificación, de continuidad y de conexión estrecha con las acciones que se trata de guiar en el mismo momento. Aplicamos esta red a la situación de un agricultor de una región francesa de gran cultivo, y acábamos considerando algunas perspectivas tratandóse de ayuda a la decisión y de investigación.

\section{ZUSAMMENFASSUNG}

Im Zusammenhang mit der strategischen Führung von KMU stellt sich folgende Schlüsselfrage: Wie lassen sich die Ideen der strategischen Vision des Unternehmers mit den getroffenen Entscheidungen verbinden? Diese Frage wird anhand des kognitiven Ansatzes zu beantworten versucht: Es geht darum zu verstehen, wie und warum ein Individuum Entscheidungen trifft. Die Entscheidungen sind massgeblich abhängig, von der Art und Weise wie der Unternehmer seine Situation interpretiert.

Zu diesem Zweck hat man den Fall eines Agrarunternehmers gewählt, weil es sich hier um eine sehr kleine Unternehmung handelt, die in der Regel nur von einer Person geführt wird. Die strategische Vision sowie die konkreten Entscheidungen stammen von der gleichen Person.

Vorab wird von folgendem Entscheidungsschema ausgegangen: Der Agrarunternehmer hat fixe Vorstellungen. Diese stammen einerseits von der strategischen Vision und der daraus abgeleiteten Unternehmungspolitik. Andererseits entspringen sie den Aktionsprogrammen, die dem Unternehmer operative, wiederkehrende Entscheide ermöglichen und somit das fortlaufende Funktionieren seiner Unternehmung sicherstellen. Die Vereinbarkeit dieser beiden Ansatzpunkte wird gewährleistet durch Entscheide in der strategischen Führung, die für unser Verständnis nichts anderes als Entscheide zur Bewältigung des Unternehmungswandels sind.

Als letzter Punkt wird ein Analysegitter um diese Enscheide konstruiert. Die Entscheide folgen gleichzeitig der Problemlösungslogik und der Uebereinstimmungslogik, dass heisst das Individuum strebt nach einer befriedigenden, vorderhand unbekannten Lösung, die sich auf seine fixen Vorstellungen abstützt. Diese beiden Logiken richten sich nach Einfachheit, Kontinuität sowie Verknüpfung von gleichzeitig auszuführenden Aktionen.

Dieses Analysegitter wurde bei einem Agrarunternehmer eines grossen französischen Kulturlandes angewendet und es wurde beabsichtigt, Hilfeleistungen für Entscheide und Forschung bieten zu können. 


\section{Introduction}

Dans le domaine de la gestion stratégique des PME, le concept de vision stratégique occupe une place croissante (par exemple, Carrière, 1991 ; Filion, 1991). Pour étudier ce concept empiriquement, les techniques de cartographie cognitive semblent une voie prometteuse (Cossette, 1996). Toutefois, comme le souligne cet auteur, une des questions clés qui demeure est de savoir comment sont reliées les idées contenues dans une vision stratégique aux décisions réelles des individus. On se propose ici d'aborder cette question par une approche cognitive de la gestion stratégique d'une PME. Par approche cognitive, on entend une approche plaçant l'analyse des comportements des acteurs au centre des études menées sur les organisations : il s'agit de comprendre pourquoi et comment les acteurs prennent leurs décisions et, en l'occurrence, leurs décisions de gestion stratégique. On choisira le cas de l'entreprise agricole pour prospecter cette voie : en effet, on a affaire ici à une très petite entreprise, généralement pilotée par un seul décideur (ou par un tandem assimilable à un décideur unique); de ce fait, c'est le même individu qui a une vision stratégique de son entreprise et qui prend les décisions concrètes qui en découlent, sans déléguer tout ou partie de celles-ci à d'autres personnes ou à des services spécialisés.

Quel point de vue adopter pour analyser une décision ? Lorsqu'on cherche à mettre en évidence la vision stratégique d'un individu pour mieux comprendre sa gestion stratégique, on considère implicitement qu'une décision procède d'une logique d'adéquation (March, 1994) : la décision permet de s'adapter à des situations en s'appuyant sur des représentations mentales qui orientent le choix. Ainsi, pour Cossette (1996), la vision stratégique, qui correspond à une façon de voir l'entreprise et son environnement ainsi qu'à des principes directeurs de gouvernement, peut être vue comme l'ensemble des représentations générales qu'un décideur peut avoir de son entreprise et qui guide ses choix. Mais une décision individuelle peut aussi être vue comme procédant d'une logique de choix (March, 1994) : dans cette optique, la décision se prend parmi un ensemble d'options à partir d'une évaluation des conséquences de celles-ci en tenant compte des préférences du décideur. Alors que la logique d'adéquation met l'accent sur la manière dont un individu utilise ses représentations pour prendre ses décisions, la logique de choix insiste sur la manière dont celui-ci traite des informations pour aboutir à une décision.

Trois mots clés demandent à être précisés : ceux de représentation mentale, d'information et de situation. La notion de « représentation mentale » relève du champ de la psychologie : elle repose sur le postulat qu'il existe dans le système cognitif humain des entités qui conservent les informations concernant les interactions de l'individu avec le monde extérieur et que celles-ci peuvent être utilisées pour des actions ultérieures (Le Ny et al., 1992); mais ces entités reflètent-elles une fraction de l'univers extérieur ou sont-elles construites par l'individu ? La notion d' « information » aussi est objet de débat : l'information est-elle donnée et ne s'agit-il que de compiler celle-ci pour trouver une solution? Ou bien, cette information doit- 
elle être recherchée et, dans ce cas, qu'est-ce qui guide cette recherche ? Enfin, la notion de « situation » est ambiguë : s'agit-il d'un ensemble de circonstances données ou de relations qui unissent un individu à son environnement à un moment donné ?

Pour notre part, nous plaçons notre approche dans le champ balayé par les deux concepts de « rationalité limitée » (Simon, 1945) et d' « enaction » (enactment) [Weick, 1969], ce qui va nous permettre de nous situer par rapport à ces questions. Les deux logiques décrites précédemment sont rationnelles dans la mesure où elles relèvent l'une et l'autre de la raison, c'est-à-dire de la faculté de penser de l'homme, en ce qu'elle permet à celui-ci de connaître et de juger, et d'appliquer son entendement à l'action. Mais, parce que l'homme vit dans un monde complexe où règne l'incertitude et du fait de ses capacités cognitives limitées, il ne peut faire preuve que d'une « rationalité limitée » (Simon, 1945) : il est amené à prendre des décisions, alors que toutes les possibilités de décisions ne sont pas connues, que toutes les conséquences ne peuvent pas être prévues et que ses préférences elles-mêmes varient et ne sont pas clairement définies; il doit s'adapter, alors que les situations auxquelles il fait face ne sont pas définies a priori, qu'il ne dispose pas de représentations permettant de traiter toutes les situations et que le processus d'adaptation, c'est-à-dire la mise en correspondance entre les représentations et les situations, n'est pas immédiat. Le concept de « rationalité limitée » nous autorise ainsi à penser que c'est la signification donnée par un décideur à telle ou telle information à un moment donné qui importe, et la notion d'interprétation devient alors centrale. L'individu joue donc un rôle actif dans la sélection des informations qu'il traite et dans les réarrangements qu'il opère sur celles-ci. C'est aussi ce que traduit le concept d' « enaction » (enactment) de Weick (1969), qui renvoie à une idée de circularité entre le sujet et l'objet. Dans ces conditions, une représentation ne peut être vue comme un simple reflet du monde environnant du décideur, mais comme une construction particulière de celui-ci; en outre, il ne peut y avoir séparation entre un « intérieur» et un « extérieur », et la notion de situation cristallise à un moment donné les relations entre un individu et son environnement.

Il en résulte trois principes de base du comportement humain :

- simplification : à un moment donné, l'individu n'accorde qu'une attention sélective aux informations de son environnement et de sa mémoire et prend ses décisions au regard d'un schéma simplifié de la situation, qui n'est pas donné mais construit par lui ; il ne recherche qu'une solution satisfaisante, qui est la meilleure solution possible dans des circonstances données ;

- continuité et orientation des décisions à travers un cadre de représentations : l'interprétation ne s'effectue pas de façon déconnectée de l'histoire de l'individu, de ses règles et de ses croyances, mais au regard d'un cadre de représentations qui lui permet d'apprécier les questions qui se posent à lui et de préciser les informations à rechercher ; 
- connexion à l'action à mener au même moment : l'interprétation est contingente des circonstances du moment et, en particulier, de l'action à mener au même moment (Varela, 1988).

Cet angle d'analyse de la décision nous conduira tout d'abord à préciser notre représentation globale des décisions dans une entreprise agricole, puis à construire une grille d'analyse des décisions de gestion stratégique. Nous appliquerons alors celle-ci à un cas particulier de gestion stratégique dans une entreprise agricole, avant d'envisager quelques perspectives de travail.

\section{La gestion stratégique dans une entreprise monodécideur}

\subsection{Représentation globale des décisions}

Il découle tout d'abord des présupposés théoriques précédents que si l'homme doit faire face à la complexité et à l'incertitude de son environnement avec des capacités cognitives limitées, il cherche toutefois à se créer des îlots de certitude afin de pouvoir agir. Il se construit ainsi des représentations qui offrent une certaine stabilité et qui jouent le rôle de réducteur de complexité (Cyert et March, 1963) et d'incertitude (Mintzberg, 1994).

Nous avons vu que la vision stratégique peut être définie comme un ensemble de représentations générales qui visent à maintenir la cohérence de l'organisation et fournissent des principes d'orientation pour l'entreprise. Dans l'exploitation agricole, ceux-ci concernent :

- la direction de l'entreprise (association père-fils, etc.);

- les activités (activité uniquement agricole ou pluriactivité ; orientation des productions [élevage ou non ; recherche de cultures à prix garanti, etc.] ; souci de valoriser la production en ayant une politique de stockage en s'engageant dans le conditionnement, la transformation ou la commercialisation [vente directe], etc.);

- le travail (embauche ou non de main-d'œuvre salariée ; politique d'autonomie ou non dans le travail);

- la politique foncière (recherche d'agrandissement par acquisition de terres, etc.);

- les modalités générales de production (intensification, politique d'irrigation ou de drainage, etc.);

- la politique financière (limitation du recours à l'emprunt, etc.). 
Mais March et Simon (1958) nous ont montré qu'un individu peut aussi isoler des zones d'action où l'incertitude est limitée ou contrôlable, lorsqu'il mène des activités d'exécution à caractère répétitif, et qu'il peut programmer les décisions concernant celles-ci; dans l'exploitation agricole, ces activités concernent le fonctionnement courant de l'entreprise (conduite des productions et organisation du travail). Dans la mesure où l'agriculteur est ici à la fois chef d'entreprise et exécutant, ou du moins participe suffisamment aux tâches agricoles pour maîtriser le champ des décisions opérationnelles, il est possible de mettre en évidence chez lui des programmes d'action qui sont des représentations particulières sur ce qu'il convient de faire ou de ne pas faire sur le plan opérationnel (Cerf et Sebillotte, 1997).

Il est donc possible d'envisager deux plans de représentations chez un agriculteur: une vision stratégique globale de son entreprise et des programmes d'action, pour conduire les activités de l'exploitation. Suivant March et Simon (1958), on considère que ces programmes d'action peuvent être en partie exécutés indépendamment les uns des autres et qu'ils ne sont reliés que d'une façon assez vague ; c'est précisément le rôle de la vision stratégique que d'assurer une certaine cohérence d'ensemble aux décisions prises par la direction d'une entreprise.

Comment, dans ces conditions, envisager la gestion stratégique ? Tout d'abord, plusieurs auteurs ont souligné que si la vision stratégique présente une certaine stabilité, elle n'est cependant pas figée, mais au contraire évolutive et interactive avec l'action (Mintzberg, 1978; Martinet, 1983). Les décisions de gestion stratégique sont alors des décisions qui permettent de traduire ou d'actualiser la vision stratégique en actions concrètes, au fur et à mesure de l'évolution des préférences de l'individu ou de l'arrivée de nouvelles informations concernant le fonctionnement d'ensemble de l'entreprise. Mais il s'agit aussi d'adapter les programmes d'action afin de remédier aux erreurs éventuelles et de résoudre des problèmes latents, ou encore afin de répondre à de nouveaux besoins, fonctions qui relèvent également de la gestion stratégique.

Suivant à la fois des auteurs de la «stratégie » comme Avenier (1988) ou du « contrôle de gestion » comme Besson et Bouquin (1991), on appelle alors décisions de pilotage stratégique ces décisions qui assurent le couplage entre vision stratégique et programmes d'action (schéma 1). Ce sont, par définition, les décisions qui gèrent le changement dans l'entreprise. Compte tenu de nos postulats de départ, nous considérons que ces décisions de changement s'ancrent dans les représentations stables de l'individu que sont la vision stratégique et les programmes d'action.

Les décisions de pilotage stratégique dont les résultats sont extériorisables à un moment donné, c'est-à-dire qui ont été appliquées, sont structurantes pour les décisions opérationnelles, au moins pour la durée du cycle de production, sauf si un besoin d'ajustement se fait sentir au cours de ce cyle. En grande culture, par exemple, le cycle de production couvre plusieurs mois et se trouve rythmé par 
SCHÉMA 1

Représentation globale des décisions dans une entreprise monodécideur

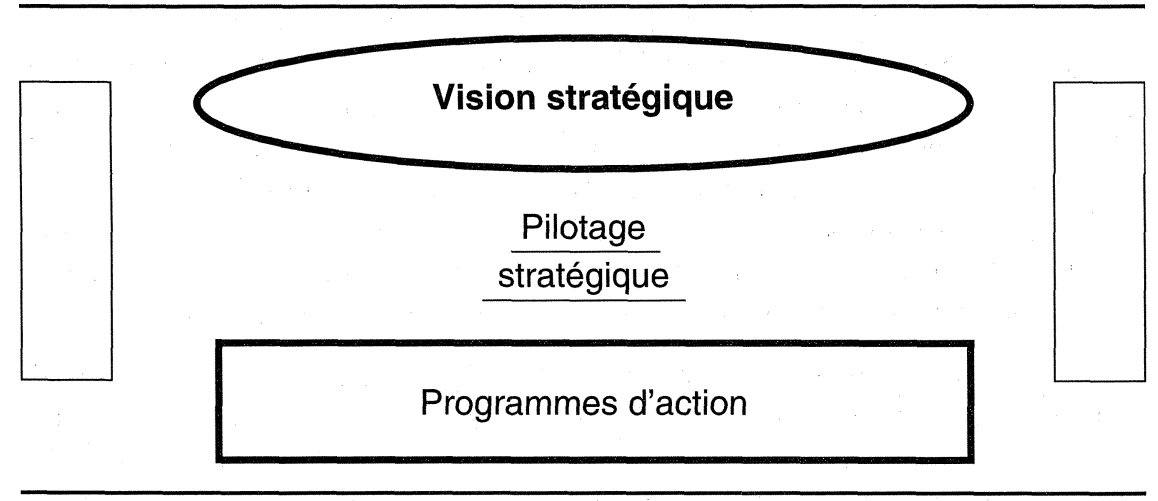

le retour des saisons (automne, pour les cultures d'hiver, printemps, pour les cultures d'été) ; les décisions de pilotage stratégique structurent donc au minimum la campagne culturale, soit une durée de l'ordre d'une année. Dans des exploitations où les cycles de production sont plus courts (maraîchage) ou plus longs (engraissement de bovins), il faudrait reconsidérer cet aspect.

Ces décisions fixent ainsi, pour la durée de la campagne, le choix précis :

- des activités (assolement, etc.);

- du cadre spatial et de son organisation (échanges de parcelles; parcelles mises en culture, etc.);

- de la main-d'œuvre et des relations de travail avec l'extérieur (recours à une entreprise pour la récolte de betterave sucrière ; recours à de la maind'œuvre occasionnelle pendant trois mois à l'automne, etc.) ;

- de la répartition des activités dans l'espace (localisation des cultures selon les terrains, etc.) et de la répartition éventuelle des tâches par personne (spécialisation de telle personne dans telle activité, etc.);

- des matériels et des équipements;

- des éléments de gestion financière.

Parmi ces décisions de pilotage stratégique, on peut a priori penser que des décisions comme l'embauche d'un salarié permanent n'ont pas le même statut que des décisions comme le recours temporaire à une personne une année donnée, par exemple.

Dès lors, comprendre la gestion stratégique dans une entreprise agricole revient à comprendre comment se prennent les décisions de pilotage stratégique à un moment donné et comment celles-ci s'articulent au cours du temps. 
Cet angle d'analyse, qui met l'accent sur les décisions de gestion stratégique, permet de dépasser, comme le soulignent Laroche et Nioche (1994), l'inconvénient des approches stratégiques « qui négligent les microprocessus pour ne considérer que les phénomènes globaux de changement stratégique » (p. 72). En effet, « celles-ci ne donnent jamais qu'un point de vue de Sirius, intéressant pour l'analyse, mais trop éloigné des acteurs. Car la réalité de l' action stratégique des dirigeants est bien celle de décisions ponctuelles, de problèmes locaux, d'actions limitées : de microprocessus » (Ibid.). Toutefois, ces mêmes auteurs insistent sur le fait qu'on ne peut pour autant « réduire le processus stratégique à un enchainement de décisions ponctuelles. On y perd la vision d'ensemble, la trame ou la continuité qui, précisément, constitue la stratégie réellement menée par l'organisation » (Ibid.).

Nous nous proposons donc maintenant de construire une grille d'analyse des décisions de pilotage stratégique qui nous permette à la fois de comprendre celles-ci individuellement et concrètement et d'en dégager le fil conducteur.

\subsection{Construction d'une grille d'analyse des décisions de pilotage stratégique}

Si nous nous intéressons au processus d'action stratégique, donc à un enchaînement de décisions de pilotage stratégique, notre unité d'analyse est la décision de pilotage stratégique elle-même, c'est-à-dire une modification élémentaire dans ce champ de décisions. En effet, dès que l'on met l'accent sur les modalités d'interprétation de l'individu dans la prise de décision, on doit revenir au moment particulier où s'est nouée la décision et comprendre ce qui s'est passé à ce moment-là : pourquoi l'individu s'est-il penché sur cette question particulière et a-t-il décidé d'agir? Pourquoi s'est-il engagé dans ce chemin particulier et a-t-il écarté d'autres voies ? Il s'agit là d'une différence importante avec les approches menées sur le changement organisationnel (Pettigrew, 1987, notamment), lesquelles cherchent à établir des relations au cours du temps entre un phénomène de changement et un contexte particulier, considéré comme donné, sans prendre en compte que ce contexte-là n'existe que du fait même des réactions spécifiques de l'organisation par rapport à certains événements à ce moment-là et que l'organisation a la possibilité de s'engager dans différentes voies.

Dans ces conditions, il convient tout d'abord de s'interroger sur la nature des décisions de pilotage stratégique. March et Simon (1958) parlent d' « activités résolutoires » pour qualifier des décisions qui ne relèvent pas d'activités répétitives d'exécution ${ }^{1}$,

1. Les activités répétitives correspondent à « une définition bien structurée de la situation qui comprendra un répertoire des schémas de réponses, et des schémas permettant la sélection d'une réponse appropriée et spécifique dans le répertoire » (March et Simon, 1958, p. 137). 
donc qui ne découlent pas de procédures ${ }^{2}$. Pour Mintzberg, Raisinghani et Théoret (1976), ce type de décision est caractérisé par le fait que, d'habitude, l'individu n'a pas de vision claire de la situation dans laquelle il est et du chemin qui conduit à la solution, et qu'il n'a qu'une vague idée de ce que la solution pourrait être. Cette description rejoint celle que font les psychologues des activités de résolution de problème, où le sujet ne dispose pas immédiatement d'une procédure pour atteindre un but ; il s'agit de situations où les données initiales s'expriment en des termes très différents des spécifications de la solution, où il existe plusieurs solutions possibles et où il s'agit de préciser le but à atteindre (Hoc, 1987).

Si l'on tient compte, par ailleurs, du rôle de couplage des décisions de pilotage stratégique entre vision stratégique et programmes d'action, on en déduit alors qu'une décision de pilotage stratégique peut être vue comme procédant de deux logiques s'articulant l'une à l'autre :

- d'une part, une logique de choix, et plus particulièrement de résolution de problème ;

- d'autre part, une logique d'adéquation, et plus particulièrement d'adaptation à des situations, en s'appuyant sur les programmes d'action et la vision stratégique.

Dans l'une et l'autre de ces deux logiques, c'est l'interprétation même de l'individu à l'œuvre au cours du processus de décision qui guide son action. Celle-ci obéit aux trois principes énoncés plus haut.

\section{Une logique de résolution de problème}

Un processus de résolution de problème peut être schématisé en deux phases, l'une de formulation du problème, qui permet de définir une situation de problème, l'autre de recherche d'une solution satisfaisante (Hoc, 1987). Il est possible de distinguer trois types de situations de problème (Mintzberg, Raisinghani et Théoret, 1976) :

- des situations d'opportunité, correspondant à la possibilité d'améliorer une situation sûre par ailleurs ;

- des situations de crise, où une impossibilité de réaliser un travail est ressentie en cours d'action et doit être traitée immédiatement, sans pouvoir disposer de délais ;

2. Les psychologues considèrent qu'une activité d'exécution est guidée par une procédure : il s'agit d'un système d'opérations dont l'exécution a pour objectif de transformer un objet en le faisant passer d'un état à un autre, et qui est soumise à une structure de contrôle visant à identifier les conditions d'exécution et à sélectionner l'ordre d'exécution des opérations (Hoc, 1987). Une procédure comporte donc un certain nombre de règles d'action, permettant d'agir dans un contexte local, jugé suffisamment stable par l'individu. 
- des situations de problème stricto sensu, entre les deux, liées à des pressions moins fortes que précédemment.

Comment un processus de résolution de problème se déroule-t-il dans le cas de situations de problème stricto sensu (Cyert et March, 1963 ; Mintzberg, Raisinghani et Théoret, 1976) ?

- La phase de formulation du problème permet de définir une situation de problème à partir d'informations de natures, a priori, diverses, globales (départ d'un salarié par exemple) ou locales (casse de matériel, par exemple) qui peuvent être ressenties ou anticipées ; ces informations sont analysées quant à leurs effets sur les actions à mener, effets à caractère négatif ici. L'individu opère une sélection des informations, du point de vue de l'étendue des effets de celles-ci (seuls certains types d'effets sont examinés et seuls les effets à certains endroits de l'exploitation sont examinés pour une information à caractère global) et du point de vue de la temporalité des effets (l'individu anticipe peu et focalise son attention sur des effets ressentis en cours d'action, sur de mauvais résultats susceptibles de se reproduire durant la campagne à venir, ou sur des effets prévus à court terme, portant sur la campagne culturale). Son diagnostic aboutit à la définition d'une situation de problème stricto sensu, lorsqu'il ressent des insatisfactions ou des menaces pour lesquelles il juge ne pas disposer d'une marge de sécurité suffisante ; il doit apporter des modifications à son organisation, et ce, avant la prochaine fois, en disposant donc d'un certain délai.

- La recherche d'une solution satisfaisante est aussi simplifiée : la recherche de solutions est séquentielle (le choix d'une solution satisfaisante résulte soit du cheminement suivi lors de la recherche des solutions et s'impose donc comme seule solution satisfaisante, soit de la comparaison de quelques solutions apparentées) et la comparaison entre les solutions envisagées ne porte que sur certains aspects. Cette recherche de solution est également marquée par la continuité, dans la mesure où elle est orientée par la formulation du problème (et notamment par sa précision et sa rapidité) et où elle s'effectue de préférence dans le voisinage des symptômes du problème et de l'ancienne solution, puis avec une recherche de complexité croissante. Le principe de connexion du raisonnement à l'action à mener au même moment se traduit par un rôle important des délais dans le processus.

Enfin, parce que l'individu se contente d'une solution satisfaisante, il sera amené à établir des priorités et à accepter des compromis dans sa décision.

Ce schéma général présente quelques variantes pour les deux autres types de situations de problème : 
- pour les situations d'occasions à saisir, la formulation du problème s'effectue nécessairement dans une perspective dynamique, puisqu'il s'agit d'améliorer l'existant, tandis que la recherche d'une solution satisfaisante est simplifiée, du fait de la préexistence d'une solution;

- pour les situations de crise, la formulation du problème comme la recherche d'une solution satisfaisante sont simplifiées du fait de la pression du temps.

À ce stade, il s' agit de comprendre ce qui fait émerger les significations (Laroche et Nioche, 1994) : comment les informations de départ sont-elles sélectionnées et évaluées? Quelles sont les solutions envisagées ? Comment les qualités d'une solution s'apprécient-elles ? Nous allons maintenant chercher à répondre à ces questions en examinant les décisions de pilotage stratégique selon une logique d'adéquation.

\section{Une logique d'adéquation}

Il s'agit ici de voir quel est plus précisément le contenu du cadre d'interprétation de l'agriculteur, sachant que celui-ci se compose des deux plans de représentations que sont la vision stratégique du décideur et ses programmes d'action.

Deux types de représentation mentale doivent être distingués selon Richard (1990), qui considère que tous les psychologues cognitivistes semblent d'accord sur cette distinction, même si les appellations diffèrent ; ce sont pour lui :

- d'une part, les « représentations » sensu stricto, qui sont des constructions circonstancielles élaborées dans un contexte particulier et à des fins précises, donc liées à des actions à mener;

- d'autre part, les « croyances », qui sont aussi des constructions, mais qui ne s'expriment pas directement sous forme de connaissances organisées en vue de l'action.

On considère alors que le cadre d'interprétation présente deux dimensions :

- le cadre organisationnel, constitué de représentations sensu stricto; ce cadre comporte les autres décisions de pilotage stratégique, ainsi que les dispositifs et les procédures d'exécution des programmes d'action organisés en différentes tâches; une tâche peut être décrite par son but (type de travail ${ }^{3}$, de production et éventuellement type de terrain), les opérations qui y sont conduites, la combinaison des moyens matériels et humains mise en œuvre et l'organisation spatiotemporelle de son déroulement ;

3. Par exemple, mise en place, fertilisation et lutte contre les ennemis des cultures, ou bien récolte pour les productions végétales. 
- les croyances, avec des croyances générales sur le plan de la vision stratégique et des croyances particulières sur le plan des programmes d'action (nécessité de labourer, par exemple).

Le cadre organisationnel précise les activités et les moyens existants à un moment donné et le réseau particulier de modalités et de contraintes à l'intérieur duquel ceux-ci s'insèrent, notamment du fait d'une différenciation possible des tâches en sous-tâches (selon les matières à « travailler », les conditions physiques du travail et les relations de travail avec l'extérieur), ou de relations possibles entre les tâches. Il permet ainsi de «localiser » le problème, c'est-à-dire de relier une information de départ avec une tâche (une sous-tâche) donnée et de préciser les limites dans lesquelles le problème est susceptible d'être raisonné ; il permet aussi de spécifier les critères de raisonnement en des termes particuliers (largeur de travail à considérer pour un achat de matériel, par exemple).

Les croyances jouent sur les effets auxquels l'agriculteur sera sensible ou peu sensible et sur la nature des solutions qu'il aura tendance à préférer ou à écarter ; elles jouent aussi sur l'interprétation dans une perspective dynamique, en permettant de rapprocher des diagnostics instantanés avec des visions de tendance, et notamment d'apprécier la pérennité des problèmes considérés et l'intérêt des occasions à saisir.

\section{Grille d'analyse}

Comment articuler ces logiques ? Nous proposons pour cela la grille d'analyse suivante :

- nous distinguons phase de formulation du problème et phase de recherche d'une solution;

- dans la phase de formulation du problème, nous précisons :

- les informations de départ auxquelles l'agriculteur a été sensible ;

- la nature de la situation de problème à laquelle il veut faire face (circonstances opportunes, crise ou problème stricto sensu) et son appréciation sur la pérennité de ce problème (problème ponctuel ou problème susceptible de se répéter);

- le cadre d'interprétation mobilisé, en distinguant ce qui relève du cadre organisationnel de ce qui relève de croyances (générales ou particulières): ce cadre permet de comprendre la nature des effets considérés, donc de repérer les simplifications apportées par l'agriculteur; de comprendre l'appréciation sur la dynamique du problème et le degré de connexion à l'action du moment ;

- dans la phase de recherche d'une solution satisfaisante, nous précisons:

- les solutions envisagées par l'agriculteur; 
- le cadre d'interprétation mobilisé, en distinguant là aussi cadre organisationnel et croyances (générales ou particulières) : ce cadre permet de comprendre la nature des solutions envisagées et des qualités recherchées, donc les simplifications apportées; de repérer le degré de continuité de celles-ci avec les solutions existantes et le rôle des délais par rapport à l'action du moment ;

- les compromis acceptés par l'agriculteur.

Le tableau 1 reprend la structure de cette grille d'analyse.

Sur le plan pratique, l'emploi de cette grille d'analyse repose sur le recueil préalable des verbalisations de l'individu, qui passe par l'utilisation d'un guide d'entretien. Celui-ci permet dans une première partie de lister les décisions de gestion stratégique prises au cours de la période étudiée et de mettre en évidence le cadre d'interprétation du décideur, notamment la vision stratégique et les programmes d'action au cours de cette période; dans une deuxième partie, les décisions sont étudiées une par une en demandant à l'individu quelles sont les raisons qui les motivent et en s'appuyant sur les données recueillies dans la première partie pour vérifier et préciser ses dires.

\section{Mise en œuvre de la grille d'analyse dans une exploitation agricole}

La grille d'analyse que nous venons de présenter a été utilisée pour comprendre les décisions d'équipement d'une quinzaine d'agriculteurs de grande culture (Allain, 1997). Afin de montrer son intérêt pour comprendre d'autres types de décision de pilotage stratégique prises par un agriculteur et leur articulation au cours du temps, nous avons choisi l'exemple d'un agriculteur de grande culture qui, en quelques années, a réorganisé l'ensemble de son parc matériel et de son travail et a modifié substantiellement son assolement, après une rupture d'association avec son frère, avec qui il entretenait des relations de travail étroites.

Nous commencerons par retracer l'histoire des décisions de pilotage stratégique avant d'appliquer la grille d'analyse.

\subsection{Chronologie des décisions de pilotage stratégique de Monsieur W.}

À la suite de l'été 1987, Monsieur W., qui travaillait en relation étroite avec son frère, lequel dirigeait une exploitation similaire (installation simultanée des deux frères en novembre 1981), doit réorganiser son exploitation à cause d'une mésentente qui a provoqué une rupture subite d'association. Son exploitation est située en Picardie dans une région de grandes cultures où l'environnement socioéconomique présente de nombreux atouts, mais dans une zone aux terrains relativement contraignants 
TABLEAU 1

Dscription et analyse des décisions de pilotage stratégique de Monsieur W.

\begin{tabular}{|c|c|c|c|c|c|c|c|}
\hline Dates & \begin{tabular}{|l|} 
Décisions \\
- assolement \\
- main-d'cuvre \\
- équipements \\
- gestion financière
\end{tabular} & $\begin{array}{l}\text { INFORMATIONS } \\
\text { DE DÉPART }\end{array}$ & $\begin{array}{l}\text { SITUATION DE PROBLÈME } \\
\text { - nature : problème, crise, } \\
\text { occasion d'affaires } \\
\text { - pérennité du problème }\end{array}$ & $\begin{array}{l}\text { CADRE D'INTERPRÉTATION } \\
\text { cadre organisationnel } \\
\text { (tâche/sous-tâche ; pilotage } \\
\text { stratégique) / croyances } \\
\text { générales - particulières }\end{array}$ & SOLUTIONS ENVISAGÉES & \begin{tabular}{|l|} 
CADRE D'INTERPRÉTATION \\
- cadre organisationnel/croyances \\
générales - particulières \\
- degré de continuité /délais
\end{tabular} & СомрRоміs \\
\hline \multirow[t]{2}{*}{ sept. 1987} & $\begin{array}{l}\text { herse alternative } \\
(4 \mathrm{~m})\end{array}$ & \multirow{2}{*}{$\begin{array}{l}\text { impossibilité de continuer à } \\
\text { utiliser les matériels du } \\
\text { frère et absence d'autres } \\
\text { matériels }\end{array}$} & \multirow[t]{2}{*}{$\begin{array}{l}\text { - crise : impossibilité d'assurer } \\
\text { le travail à venir } \\
\text { - problème durable }\end{array}$} & \multirow{2}{*}{$\begin{array}{l}\text { - sous-tâche: mise en place / } \\
\text { céréales d'hiver } \\
\text { - mésentente durable }\end{array}$} & \multirow{2}{*}{$\begin{array}{l}\text { - achat de matériels } \\
\text { - mêmes matériels que le } \\
\text { frère }\end{array}$} & \multirow{2}{*}{$\begin{array}{l}\text { - autonomie dans le travail } \\
\text { - organisation de la tâche } \\
\text { jusqu'alors } \\
\text { - solution à trouver vite } \\
\text { - gestion financière: situation } \\
\text { tendue }\end{array}$} & - \\
\hline & $\begin{array}{l}\text { semoir à céréales } \\
(4 \mathrm{~m})\end{array}$ & & & & & & semoir acheté d'occasion \\
\hline \multirow[t]{2}{*}{ oct. 1987} & $\begin{array}{l}\text { tracteur } \\
(150 \mathrm{CV})\end{array}$ & $\begin{array}{l}\text { impossibilité de continuer à } \\
\text { utiliser le tracteur du frère }\end{array}$ & $\begin{array}{l}\text { - crise : pas de tracteur assez } \\
\text { puissant pour le travail à venir } \\
\text { - problème durable }\end{array}$ & $\begin{array}{l}\text { - ensemble des tâches, mais } \\
\text { surtout celles d'automne } \\
\text { - tracteurs existants ( } 2 \text { de } 90 \text { - } \\
95 \mathrm{CV} \text { ) } \\
\text { - } \text { mésentente durable }\end{array}$ & $\begin{array}{l}\text { - un tracteur plus puissant } \\
\text { pour créer une réserve de } \\
\text { puissanœ } \\
\text { - revente du tracteur le plus } \\
\text { usé }\end{array}$ & $\begin{array}{l}\text { - tracteurs existants } \\
\text { - tracteur utilisé par le frère } \\
(100 \mathrm{CV}) \\
\text { - solution à trouver vite } \\
\text { - gestion financière: situation } \\
\text { tendue }\end{array}$ & $\begin{array}{l}\text { mauvaise réputation, mais } \\
\text { bonne occasion; achat en } \\
\text { leasing }\end{array}$ \\
\hline & charrue (6 corps) & $\begin{array}{l}\text { impossibilité de maintenir } 2 \\
\text { postes de travail }\end{array}$ & $\begin{array}{l}\text { - problème : vitesse de travail } \\
\text { insuffisante } \\
\text { - problème durable }\end{array}$ & $\begin{array}{l}\text { - sous-tâche : mise en place / } \\
\text { céréales d'hiver / derrière } \\
\text { betteraves } \\
\text { - mésentente durable }\end{array}$ & $\begin{array}{l}\text { - une charrue plus large } \\
\text { pour labourer en un poste } \\
\text { (+ limitation des labours) } \\
\text { - revente de la charrue } \\
\text { présente }\end{array}$ & $\begin{array}{l}\text { - charrue existante ( } 4 \text { corps) } \\
\text { - charrue utilisée par le frère } \\
\text { ( } 5 \text { corps) } \\
\text { - gestion financière : situation } \\
\text { tendue }\end{array}$ & - \\
\hline fév. 1988 & $\begin{array}{l}\text { licenciement d'un } \\
\text { salarié }\end{array}$ & $\begin{array}{l}\text { un demi-temps de salarié en } \\
\text { plus }\end{array}$ & $\begin{array}{l}\text { - problème : coût trop élevé des } \\
\text { charges salariales } \\
\text { - problème durable }\end{array}$ & \begin{tabular}{|l|} 
gestion financière: situation \\
tendue (emprunt \\
d'installation à rembourser)
\end{tabular} & licenciement du salarié & $\begin{array}{l}\text { - simplification du travail } \\
\text { - gestion financière : situation } \\
\text { tendue }\end{array}$ & $\begin{array}{l}\text { moins grande souplesse } \\
\text { dans le travail }\end{array}$ \\
\hline $\begin{array}{l}\text { printemps } \\
1988\end{array}$ & \begin{tabular}{|l|} 
- emprunt $(500000 \mathrm{~F}$ \\
sur 7 ans)
\end{tabular} & dépenses à effectuer & $\begin{array}{l}\text { - problème : impossibilité de } \\
\text { financer les investissements }\end{array}$ & \begin{tabular}{|l|} 
gestion financière : situation \\
tendue (emprunt \\
d'installation à rembourser)
\end{tabular} & recours à un emprunt & $\begin{array}{l}\text { gestion financière : } \\
\text { capacité de remboursement } \\
\text { d'emprunt }\end{array}$ & $\begin{array}{l}\text { montant limité de } \\
\text { l'emprunt }\end{array}$ \\
\hline $\begin{array}{l}\text { printemps } \\
1988\end{array}$ & \begin{tabular}{|l|}
$\begin{array}{l}\text { arrêt des céréales à } \\
\text { paille (sauf blé) }\end{array}$ \\
forte réduction du \\
maïs grain
\end{tabular} & suppression d'un salarié & $\begin{array}{l}\text { - problème : charge en travail } \\
\text { - très élevée } \\
\text { - problème durable }\end{array}$ & - simlification du travail & $\begin{array}{l}\text { - diminution : } \\
\cdot \text { du nombre de cultures } \\
\cdot \text { des cultures } \\
\text { consommatrices en travail } \\
\text { (maïs) }\end{array}$ & simplification du travail & \\
\hline & $\begin{array}{l}\text { introduction de } 15 \\
\text { chevaux en pension }\end{array}$ & & & & - de la surface cultivée & - goût pour l'élevage des chevaux & - \\
\hline
\end{tabular}


TABlEaU 1 (suite)

Dscription et analyse des décisions de pilotage stratégique de Monsieur W.

\begin{tabular}{|c|c|c|c|c|c|c|c|}
\hline & $\begin{array}{l}\text { introduction de } \\
10 \text { ha de cultures } \\
\text { fourragères }\end{array}$ & \multirow{4}{*}{ proposition d'un voisin } & & \multirow[t]{2}{*}{$\begin{array}{l}\text { - assolement et utilisation } \\
\text { des terrains }\end{array}$} & \multirow{2}{*}{$\begin{array}{l}\text { - chevaux en pension } \\
\text { (nourris par fourrages) } \\
\text { - mise en location de terres }\end{array}$} & \multirow[t]{2}{*}{$\begin{array}{l}\text { + idée d'ouvrir un centre } \\
\text { hippique à terme }\end{array}$} & \\
\hline & \begin{tabular}{|l|} 
location de 5 ha à \\
un autre \\
agriculteur \\
\end{tabular} & & $\begin{array}{l}\text { - occasion : possibilité de } \\
\text { louer des terres à un voisin }\end{array}$ & & & & \\
\hline mars 1988 & \begin{tabular}{|l|} 
agrandissement du \\
Marsk-sem
\end{tabular} & & \multirow{2}{*}{$\begin{array}{l}\text { - problème : vitesse de travail } \\
\text { insuffisante } \\
\text { - problème durable }\end{array}$} & \multirow{2}{*}{$\begin{array}{l}\text { - sous-tâche : mise en place } \\
\text { /betteraves sucrières } \\
\text { - simplification du travail } \\
\text { - politique d'insatisfaciton } \\
\end{array}$} & \multirow{2}{*}{$\begin{array}{l}\text { préparation du sol en un } \\
\text { passage avec un outil } \\
\text { combiné (Germador) + un } \\
\text { outil regroupé (agrandi) }\end{array}$} & \multirow{2}{*}{$\begin{array}{l}\text { - organisation de la tâche } \\
\text { jusqu'alors } \\
\text { - nouveau tracteur } \\
\text { - simplification du travail } \\
\end{array}$} & - \\
\hline avril 1988 & $\begin{array}{l}\text { construction du } \\
\text { Germador }(6 \mathrm{~m})\end{array}$ & & & & & & $\begin{array}{l}\text { - autoconstruction pour } \\
\text { limiter le coût }\end{array}$ \\
\hline été 1989 & $\begin{array}{l}\text { moisson par } \\
\text { entreprise }\end{array}$ & $\begin{array}{l}\text { Id. + expérience de la } \\
\text { moisson } 1988 \text { à deux }\end{array}$ & $\begin{array}{l}\text { - problème : pénibilité + } \\
\text { manque de vitesse } \\
\text { - problème durable }\end{array}$ & \multirow{3}{*}{$\begin{array}{l}\text { - sous-tâches : récolte / blé } \\
\text { + pois } \\
\text { - simplification du travail }\end{array}$} & recours à une entreprise & solution adoptée par le frère & $\begin{array}{l}\text { perte d'autonomie dans le } \\
\text { travail }\end{array}$ \\
\hline oct. 1989 & $\begin{array}{l}\text { revente de la } \\
\text { moissonneuse }\end{array}$ & \multirow[t]{2}{*}{$\begin{array}{l}\text { recours à une entreprise } \\
\text { pour la moisson }\end{array}$} & $\begin{array}{l}\text { - problème : matériel inutile } \\
\text { (sauf pour maîs) } \\
\text { - problème durable }\end{array}$ & & revente de la moissonneuse & $\begin{array}{l}\text { gestion financière : situation } \\
\text { tendue }\end{array}$ & $\begin{array}{l}\text { plus de matériel pour } \\
\text { récolter le maïs grain }\end{array}$ \\
\hline janv. 1990 & benne (14t) & & $\begin{array}{l}\text { - problème : vitesse de travail } \\
\text { insuffisante } \\
\text { - problème durable }\end{array}$ & & $\begin{array}{l}\text { une benne de plus grande } \\
\text { capacité pour mieux } \\
\text { équilibrer le chantier }\end{array}$ & $\begin{array}{l}\text { - sous-tâches : récolte / blé + pois } \\
\text { - benne existante }(10 \mathrm{t})\end{array}$ & - \\
\hline \multirow[t]{4}{*}{$\begin{array}{l}\text { printemps } \\
1990\end{array}$} & arrêt du maïs grain & \multirow[t]{2}{*}{$\begin{array}{l}\text { revente de la moisson- } \\
\text { neuse-batteuse }\end{array}$} & \multirow{2}{*}{$\begin{array}{l}\text { problème : impossibilité de } \\
\text { récolter le maïs grain } \\
\text { + faible rentabilité de la culture } \\
\text { (faibles rendements dans certains } \\
\text { terrains + prix de vente élevé) }\end{array}$} & \multirow[t]{2}{*}{$\begin{array}{l}\text { sous-tâche : récolte / maïs } \\
\text { grain (terrains sableux) } \\
\text { résultats économiques }\end{array}$} & abandon du maiis grain & $\begin{array}{l}\text { - simplification du travail } \\
\text { - récolte par entreprise trop } \\
\text { coûteuse }\end{array}$ & - \\
\hline & \begin{tabular}{|l} 
introduction du \\
tournesol
\end{tabular} & & & & $\begin{array}{l}\text { une culture convenant aux } \\
\text { terres sableuses }\end{array}$ & $\begin{array}{l}\text { - moyens existants } \\
\text { - organisation du travail }\end{array}$ & - \\
\hline & $\begin{array}{l}\text { développement du } \\
\text { pois protéagineux }\end{array}$ & $\begin{array}{l}\text { prix intéressant du pois } \\
\text { protéagineux }\end{array}$ & opportunité : marché intéressant & $\begin{array}{l}\text { assolement existant }+ \\
\text { modifications de surfaces }\end{array}$ & développer le pois & $\begin{array}{l}\text { assolement existant }+ \\
\text { modifications de surfaces }\end{array}$ & - \\
\hline & $\begin{array}{l}\text { semis du toumesol } \\
\text { par entreprise }\end{array}$ & introduction du tournesol & $\begin{array}{l}\text { - problème : impossibilité } \\
\text { d'assurer le travail }\end{array}$ & $\begin{array}{l}\text { sous-tâche : mise en place } \\
\text { (semis)/toumesol }\end{array}$ & recours à une entreprise & $\begin{array}{l}\text { solution déjà essayée (récolte) + } \\
\text { entreprise connue }\end{array}$ & - \\
\hline $\begin{array}{l}15 \text { sept.-15 } \\
\text { oct. } 1990\end{array}$ & $\begin{array}{l}\text { main-d'euvre } \\
\text { occasionnelle }\end{array}$ & \multirow[t]{2}{*}{$\begin{array}{l}\text { départ du salarié en juin } \\
1990\end{array}$} & \multirow[t]{2}{*}{$\begin{array}{l}\text { - problème durable } \\
\text { - crise : impossibilité de réaliser } \\
\text { la récolte de betteraves seul }\end{array}$} & \multirow[t]{2}{*}{$\begin{array}{l}\text { ensemble des tâches, mais } \\
\text { surtout sous-tâche : récoltel } \\
\text { betteraves sucrières }\end{array}$} & \multirow[t]{2}{*}{$\begin{array}{l}\text { rembauche d'un salarié + } \\
\text { recours à une main-d'œuvre } \\
\text { occasionnelle en attendant }\end{array}$} & \multirow[t]{2}{*}{$\begin{array}{l}\text { avoir un salarié } \\
\text { sous-tâche : récolte / betteraves } \\
\text { sucrières }\end{array}$} & \multirow[t]{2}{*}{$\begin{array}{l}\text { pas de main-d'œuvre } \\
\text { occasionnelle pour la } \\
\text { moisson/travail possible à } \\
2 \text { (avec l'entreprise) }\end{array}$} \\
\hline nov. 1990 & $\begin{array}{l}\text { embauche d'un } \\
\text { nouveau salarié }\end{array}$ & & & & & & \\
\hline
\end{tabular}


TABLEAU 1 (suite)

Description et analyse des décisions de pilotage stratégique de Monsieur W.

\begin{tabular}{|c|c|c|c|c|c|c|c|}
\hline \multirow[t]{2}{*}{$\begin{array}{l}\text { printemps } \\
1992\end{array}$} & $\begin{array}{l}\text { semis de betteraves } \\
\text { par entreprise }\end{array}$ & \multirow[t]{3}{*}{$\begin{array}{l}\text { - insatisfactions quant aux } \\
\text { résultats techniques du } \\
\text { travail }\end{array}$} & $\begin{array}{l}\text { problème : mauvaise qualité du } \\
\text { semis } \\
+ \text { semoir usé }\end{array}$ & $\begin{array}{l}\text { - sous-tâche : mise en place } \\
\text { (semis)/betteraves } \\
\text { politique d'intensification }\end{array}$ & recours à une entreprise & $\begin{array}{l}\text { - solution déjà essayée pour le } \\
\text { semis + entreprise connue } \\
\text { - un poste assuré }\end{array}$ & - \\
\hline & $\begin{array}{l}\text { rachat demi-part du } \\
\text { pulvérisateur }\end{array}$ & & $\begin{array}{l}\text { problème : manque de } \\
\text { disponibilité du pulvérisateur } \\
\text { (partage avec le frère) }\end{array}$ & $\begin{array}{l}\text { - tâche : traitements / } \\
\text { printemps } \\
\text { - politique d'intensification }\end{array}$ & $\begin{array}{l}\text { rachat de la demi-part du } \\
\text { pulvérisateur détenu en } \\
\text { copropriété avec le frère }\end{array}$ & $\begin{array}{l}\text { - organisation de la tâche } \\
\text { jusqu'alors } \\
\text { - gestion financière : situation } \\
\text { tendue }\end{array}$ & $\begin{array}{l}\text { impossibilité de continuer } \\
\text { à employer le tracteur du } \\
\text { frère, attelé jusqu'ici à } \\
\text { l'appareil, d'où } \\
\text { concurrences sur les } \\
\text { tracteurs }\end{array}$ \\
\hline mai 1992 & \begin{tabular}{|l|}
$\begin{array}{l}\text { licenciement du } \\
\text { salarié }\end{array}$ \\
\end{tabular} & & $\begin{array}{l}\text { - problème : incompétence du } \\
\text { salarié } \\
\end{array}$ & politique d'intensification & licenciement du salarié & politique d'intensification & $\begin{array}{l}\text { - risque de se retrouver } \\
\text { seul quelques mois }\end{array}$ \\
\hline été 1992 & $\begin{array}{l}\text { main-d'auvre } \\
\text { occasionnelle }\end{array}$ & \multirow[t]{2}{*}{ licenciement du salarié } & \multirow{2}{*}{$\begin{array}{l}\text { problème : absence de salarié } \\
\text { (durable) + avoir de l'aide pour } \\
\text { la récolte à venir (ponctuel) }\end{array}$} & \multirow[t]{2}{*}{$\begin{array}{l}\text { ensemble des tâches, } \\
\text { mais surtout moisson }\end{array}$} & \multirow{2}{*}{$\begin{array}{l}\text { rembauche d'un salarié + } \\
\text { recours à une main- } \\
\text { d'œuvre occasionnelle en } \\
\text { attendant }\end{array}$} & - avoir un salarié & - \\
\hline \multirow[t]{3}{*}{ sept. 1992} & $\begin{array}{l}\text { arrivée du nouveau } \\
\text { salarié }\end{array}$ & & & & & - sous-tâches : récolte / blé + pois & - \\
\hline & $\begin{array}{l}\text { nouvelle charrue } \\
\text { (5 corps) }\end{array}$ & \multirow[t]{2}{*}{$\begin{array}{l}\text { insatisfactions quant aux } \\
\text { résultats techniques du } \\
\text { travail }\end{array}$} & $\begin{array}{l}\text { - problème : maniabilité } \\
\text { insuffisante } \\
\text { - opportunité : meilleure } \\
\text { situation financière }\end{array}$ & $\begin{array}{l}\text { - tâches de mise en place } \\
\text { (labours) } \\
\text { - gestion financière : fin de } \\
\text { remboursement d'emprunt }\end{array}$ & $\begin{array}{l}\text { une charrue plus maniable } \\
\text { et suffisamment large }\end{array}$ & $\begin{array}{l}\text { - terrains contraignants } \\
\text { - tâches de mise en place } \\
\text { - charrue existante } \\
\text { - charrue du frère }(5 \text { corps })\end{array}$ & $\begin{array}{l}\text { - une charrue moins } \\
\text { large, mais plus } \\
\text { maniable }\end{array}$ \\
\hline & $\begin{array}{l}\text { cover crop } \\
\text { (36 disques) }\end{array}$ & & $\begin{array}{l}\text { - problème : vitesse de travail } \\
\text { insuffisante } \\
\text { opportunité : meilleure } \\
\text { situation financière }\end{array}$ & $\begin{array}{l}\text { - sous-tâche : mise en place / } \\
\text { blé d'hiver (sans labour) } \\
\text { - simplification du travail } \\
\text { - gestion financière : fin de } \\
\text { remboursement d'emprunt }\end{array}$ & $\begin{array}{l}\text { un matériel travaillant plus } \\
\text { vite }\end{array}$ & $\begin{array}{l}\text { - sous-tâche : mise en place / blé } \\
\text { d'hiver } \\
\text { - matériel existant }\end{array}$ & - \\
\hline fév: 1993 & $\begin{array}{l}\text { pulvérisateur } \\
\text { automoteur }\end{array}$ & proposition d'un vendeur & $\begin{array}{l}\text { - opportunité: bonne occasion } \\
\text { - problème : concurrences dans } \\
\text { le travail (tracteurs) + usure du } \\
\text { pulvérisateur }\end{array}$ & $\begin{array}{l}\text { - ensemble des tâches au } \\
\text { printemps } \\
\text { - politique d'identification - } \\
\text { gestion financière : fin de } \\
\text { remboursement d'emprunt }\end{array}$ & un pulvérisateur automoteur & ensemble des tâches / printemps & achat d'occasion \\
\hline \multirow[t]{3}{*}{$\begin{array}{l}\text { printemps } \\
1993\end{array}$} & $\begin{array}{l}\text { réduction de pois } \\
\text { protéagineux }\end{array}$ & \multirow{2}{*}{\begin{tabular}{|l} 
- insatisfaction sur les \\
résultats économiques \\
de ces cultures \\
nouvelle PAC
\end{tabular}} & \multirow{2}{*}{$\begin{array}{l}\text { problèmes : moindre rentabilité } \\
+ \text { coûts élevés } \\
\text { du travail en entreprises pour ces } \\
\text { cultures }\end{array}$} & \multirow{3}{*}{$\begin{array}{l}\text { - résultats économiques } \\
\text { - simplification du travail }\end{array}$} & \multirow[t]{2}{*}{ réduction de ces cultures } & \multirow[t]{2}{*}{ simplification du travail } & - \\
\hline & $\begin{array}{l}\text { réduction de } \\
\text { tournesol }\end{array}$ & & & & & & - \\
\hline & $\begin{array}{l}\text { augmentation de la } \\
\text { location }(10 \mathrm{ha})\end{array}$ & proposition d'un voisin & $\begin{array}{l}\text { - opportunité : possibilité de } \\
\text { louer des terres à un voisin }\end{array}$ & & augmentation de la location & - solution déjà essayée & - \\
\hline
\end{tabular}


du fait de leur hétérogénéité. Elle a une surface moyenne (165 ha) et est orientée vers les productions végétales ( 45 ha de betteraves sucrières, 70 ha de blé et autres céréales à paille, 35 ha de maïs grain et 15 ha de pois protéagineux). Elle dispose de deux salariés, dont l'un travaillait quasiment à mi-temps sur l'exploitation du frère (celui-ci n'embauchait qu'un seul salarié), et les deux salariés se retrouvent donc à plein temps chez Monsieur W.

Le tableau 1 retrace, dans les deux colonnes de gauche, la chronologie des 33 décisions de pilotage stratégique prises par l'agriculteur au cours de la période de réorganisation qui dure approximativement cinq ans et demi :

- à l'automne 1987, il achète divers instruments de travail du sol et de semis (une herse alternative se combinant au semoir à céréales ; une charrue plus large) et un tracteur plus puissant;

- au printemps 1988, il licencie l'un des deux salariés et change son assolement (il arrête la production des céréales à pailles autres que le blé et réduit fortement la part de maïs grain, tandis qu'il introduit une production de cultures fourragères sur une partie des surfaces libérées [10 ha] et loue le reste [5 ha] à un autre agriculteur); il prend également une quinzaine de chevaux en pension à partir de cette époque ; enfin, il agrandit un autre matériel de travail du sol (Marsk-sem) et en fait construire un à son salarié (Germador); sur le plan financier, il a recours à un emprunt de $500000 \mathrm{~F}$, à rembourser sur sept ans ;

- à partir de l'été 1989, il fait appel à une entreprise de travaux agricoles pour la moisson; il revend sa moissonneuse-batteuse à l'automne suivant et achète une benne de plus grande capacité au cours de l'hiver;

- au printemps 1990, il arrête définitivement la production du maïs grain, la remplace par le tournesol et développe le pois protéagineux ; il a recours à une entreprise de travaux agricoles pour le semis de tournesol ;

- en juin 1990, le salarié restant quitte l'exploitation et n'est remplacé qu'en novembre 1990; l'agriculteur emploie temporairement une personne du 15 septembre au 15 octobre;

- à partir du printemps 1992, le semis de betteraves sucrières est réalisé par une entreprise, tandis que l'agriculteur rachète la demi-part du pulvérisateur détenue par son frère ; en mai 1992, il licencie le nouveau salarié qui n'est remplacé qu'en septembre 1992 ; l'agriculteur fait à nouveau appel à de la main-d'œuvre occasionnelle, pendant l'été cette fois-ci ;

- à l'automne 1992, il modifie encore une fois son matériel de travail du sol (cover crop, charrue); en particulier, la charrue qu'il avait pourtant déjà changée à l'automne 1987 est remplacée par une charrue moins large ; 
- enfin, au printemps suivant, il remplace son pulvérisateur par un pulvérisateur automoteur ; il diminue aussi fortement les surfaces cultivées en pois protéagineux et en tournesol et remplace celles-ci par une augmentation des terres en location (10 ha).

Retracées de cette façon, les décisions prises par Monsieur W. semblent assez incohérentes :

- sur le plan de l'assolement, si l'agriculteur semble se détourner de la production de céréales pour développer la production d'oléo-protéagineux (tournesol et pois), cette orientation n'est pas maintenue ultérieurement ; en outre, le choix de prendre des chevaux en pension n'est pas habituel dans ce type d'exploitation orienté exclusivement vers les grandes cultures;

- sur le plan du travail, on peut a priori comprendre que Monsieur W. prenne la décision de licencier l'un de ses deux salariés, du fait de la réduction globale de la charge de travail; on comprend moins les changements successifs de salarié et surtout les époques variables d'embauche de maind'œuvre occasionnelle lorsque l'agriculteur se retrouve temporairement sans salarié ;

- sur le plan des investissements en matériel, enfin, il semble que MonsieurW. cherche à augmenter sa vitesse de travail par des choix appropriés (augmentation de la taille de l'outillage, etc.), mais il y a au moins une contradiction apparente dans ces choix (deuxième achat de charrue); par ailleurs, il opte pour des types différents de pulvérisateur à moins d'une année d'écart; enfin, et surtout, le recours à une entreprise de travaux agricoles pour la moisson et le semis de betteraves (alors que l'agriculteur disposait du matériel nécessaire à la réalisation de ces travaux) ne s'explique pas, ni le choix d'appliquer cette solution plutôt qu'une autre au semis de tournesol.

\subsection{Explication de ces décisions}

Une enquête approfondie auprès de Monsieur W., à l'aide d'un guide d'entretien permettant de mettre à jour les éléments de la grille d'analyse, peut expliquer ses décisions, comme nous le montre le tableau 1.

La vision stratégique de cet agriculteur s'organise autour des deux objectifs suivants : obtenir un bon revenu tout en se ménageant du temps libre. Elle s'appuie sur les idées clés de maintenir une politique d'intensification des productions - c'est-à-dire de recherche de rendements élevés -, de ne pas travailler seul mais d'avoir un salarié "pour ne pas être toujours sur le tracteur », et d'être autonome dans le travail; si l'exploitation est traditionnellement orientée vers les productions végétales de 
grande culture, Monsieur W. n'exclut pas de mieux valoriser sa localisation dans un territoire périurbain en développant des activités extra-agricoles (en s'appuyant notamment sur son goût pour les chevaux).

À l'automne 1987, l'agriculteur doit faire face à une situation de crise pour assurer la semence des céréales d'hiver : en effet, il bénéficiait jusqu'alors pour ce travail d'outils ${ }^{4}$ de son frère et il ne possède pas d'équipements qui puissent convenir ; aussi décide-t-il d'investir et il opte pour les mêmes outils que ceux de son frère. En outre, Monsieur W. ne peut plus disposer du tracteur de son frère pour tirer ces outils et n'a pas de tracteur suffisamment puissant sur son exploitation pour se charger du travail ; ce problème concerne non seulement la traction de ces outils à l'automne, mais aussi d'autres outils et d'autres époques de travail. Aussi l'agriculteur décide-t-il en quelque sorte de se créer une réserve de puissance et de remplacer l'un de ses tracteurs par un tracteur plus puissant ; il hésite entre différentes marques et différents types de tracteur et opte pour celui qui, bien qu'ayant mauvaise réputation dans son secteur, présente une puissance élevée et peut être obtenu à un prix intéressant (tracteur de démonstration). Enfin, toujours à l'automne, mais de façon moins grave, se pose le problème que les labours ne peuvent plus être menés en deux postes comme auparavant; le problème se fait surtout sentir pour les labours qui s'effectuent entre la récolte des dernières betteraves et les semis de céréales, car il faut aller vite pour semer les blés d'hiver dans de bonnes conditions et ne pas risquer d'altérer le potentiel de rendement. L'agriculteur décide donc de remplacer sa charrue par un matériel nettement plus large, tout en s'efforçant de limiter le plus possible les labours pour cette culture. Monsieur W., qui se trouve alors dans une situation financière tendue parce qu'il doit rembourser son prêt d'installation, a recours à une solution de leasing pour l'acquisition du tracteur et à un achat d'occasion pour le semoir ; les autres matériels sont achetés neufs, parce qu'ils ne peuvent être trouvés d'occasion.

Au printemps 1988, l'agriculteur licencie l'un de ses deux salariés et n'en garde qu'un seul, car il ne peut supporter des charges en personnel aussi élevées, du fait notamment du niveau d'endettement élevé de l'exploitation. Cette situation financière tendue est liée au remboursement du prêt d'installation de l'agriculteur, mais aussi au nouvel emprunt contracté par celui-ci à cette même époque pour financer la réorganisation de son exploitation. La réduction de personnel l'amène à adopter une politique de simplification du travail, qui passe :

- d'une part, par une simplification de l'assolement : l'agriculteur diminue le nombre de cultures (abandon des céréales à paille autres que le blé d'hiver) et réduit fortement le maïs grain, culture exigeante en travail ; il saisit l'offre que lui fait un voisin de louer quelques hectares et réduit donc

4. Herse alternative combinée au semoir à céréales. 
même sa surface cultivée totale ; enfin, il décide de prendre des chevaux en pension, dont il assure lui-même l'alimentation (cultures fourragères), pour, éventuellement, ouvrir à terme un centre hippique ;

- d'autre part, par une simplification des tâches de printemps, et notamment de la mise en place des betteraves sucrières qui nécessite plusieurs opérations : il s'agit d'essayer de préparer le sol en un seul passage en regroupant toutes les opérations; l'agriculteur fait ainsi construire à son salarié un matériel combiné de travail du sol, le Germador, en s'inspirant d'un modèle commercial, et attèle un outil qu'il utilisait déjà, à l'avant du tracteur tirant le Germador, ce qui nécessite un agrandissement de cet outil ; ces modifications sont permises par le tracteur plus puissant acheté à l'automne ; le choix d'autoconstruire un outil plutôt que d'acheter un matériel existant vise à limiter les dépenses.

Pour la moisson 1988, Monsieur W. se retrouve seul avec son salarié, alors qu' auparavant, la moisson s'effectuait en cinq postes ${ }^{5}$; 1 'agriculteur conduit alors sa moissonneuse-batteuse, tandis que son salarié effectue le transport. Peu satisfait de cette solution, il décide de faire appel à une entreprise de travaux agricoles dès l'été d'après, suivant en cela l'exemple de son frère qui a adopté ce système ; il revend alors sa machine à l'automne 1989, après la récolte du maïs grain, ce qui lui permet de financer le remplacement de sa benne par une benne de plus forte capacité qui équilibre mieux ce chantier.

La revente de la moissonneuse-batteuse, qui disposait d'options permettant la récolte du maïs grain, conduit l'agriculteur à abandonner cette culture déjà peu rentable (coût élevé de la récolte par entreprise pour le maïs grain) et à remplacer celle-ci par du tournesol dès le printemps suivant. Il s'agit en effet d'une culture bien adaptée à une partie de ces terrains (terres sableuses), pour lesquels il cherchait une solution satisfaisante. Il développe aussi le pois, profitant du prix intéressant de cette culture à ce moment-là. Dans la mesure où Monsieur W. n'a pas de semoir lui permettant de semer le tournesol, il décide de faire appel à l'entreprise de travaux agricoles qu'il emploie déjà pour ce travail, car il est satisfait de cette solution.

À partir de juin 1990, l'agriculteur se retrouve seul, en raison du départ de son salarié ; il doit en particulier assurer seul la moisson avec l'entrepreneur, parce qu'il ne trouve personne pour l'aider, et il embauche temporairement quelqu'un pour la récolte des betteraves sucrières, jusqu'à l'arrivée d'un nouveau salarié en novembre 1990. Monsieur W. n'envisage en effet à aucun moment de se passer définitivement de personnel.

5. Deux moissonneuses-batteuses provenant chacune de l'une des deux exploitations et conduites par les deux chefs d'exploitation, le transport étant assuré par les trois salariés. 
Or, le nouveau salarié s'avère peu compétent, ce qui vient renforcer plusieurs insatisfactions de l'agriculteur à l'égard d'une politique d'intensification, en rapport avec les tâches de printemps :

- lors de la mise en place des betteraves sucrières, il lui est impossible de surveiller en cours de travail la qualité du semis, faute de main-d'œuvre suffisante ; et le semoir, qui commence à être usé, n'effectue pas un travail très satisfaisant ; aussi l'agriculteur décide-t-il de faire également appel à une entreprise de travaux agricoles pour les semis de betteraves sucrières, et il choisit celle qu'il emploie déjà et dont il a pu apprécier la qualité du travail en matière de semis. En effet, l'opération de semis est l'une des opérations culturales les plus sensibles et, pour la betterave sucrière, la réussite du semis conditionne même directement le rendement final, donc il s'agit d'être vigilant;

- en outre, l'agriculteur continuait jusqu' alors à réaliser ses traitements avec le pulvérisateur acheté en commun avec son frère et ne pouvait donc pas disposer à tout moment de l'appareil, alors que la date d'intervention est cruciale pour cette opération ; c'est pourquoi il rachète la demi-part de son frère pour être plus autonome. Cette solution peu coûteuse présente toutefois l'inconvénient pour l'agriculteur de ne plus pouvoir lui permettre d'utiliser le tracteur de son frère, attelé jusqu'alors en permanence au pulvérisateur, donc, de l'obliger à dételer l'un de ses deux tracteurs mobilisés dans les opérations de mise en place, ce qui ralentit le travail.

Monsieur W. licencie son salarié en mai 1992. En attendant d'en retrouver un qui lui donne satisfaction, il embauche temporairement une personne pour l'aider avec la moisson afin de ne pas se retrouver seul comme à l'été 1990 ; un nouveau salarié arrive en septembre 1992.

À l'automne 1992, l'agriculteur cherche encore à accroître son efficacité dans le travail : il change sa charrue pour une charrue moins large, mais beaucoup plus maniable, donc mieux adaptée à la forme irrégulière de ses parcelles; il choisit un matériel de même largeur que celui de son frère, qui paraît donner satisfaction. Monsieur W. achète aussi un outil de travail du sol (cover crop) permettant de travailler plus vite que celui qu'il utilisait pour la mise en place des blés d'hiver sans labour. Ces acquisitions sont rendues possibles par une amélioration de la situation financière de l'agriculteur à partir de 1992 (fin de remboursement du prêt d'installation en janvier 1991 et fin du leasing sur le tracteur en décembre 1991).

Au printemps suivant, il fait l'acquisition d'un pulvérisateur automoteur, un matériel d'occasion en bon état, ce qui lui permet de supprimer les problèmes de concurrence pour les tracteurs au printemps et de disposer d'un outil plus performant et en meilleur état que son pulvérisateur qui commençait à être usé ; là encore, l'agriculteur profite de l'amélioration de sa situation financière. Cette année-là, qui 
coïncide avec l'entrée en vigueur de la nouvelle Politique agricole commune, il remodifie aussi son assolement : il réduit les surfaces cultivées en pois et en tournesol pour tenir compte à la fois de la baisse de prix de ces cultures et du coût élevé du travail en entreprise pour celles-ci ; il en profite pour augmenter la surface louée à son voisin, toujours demandeur de terrains.

\subsection{Analyse de ces décisions}

Comprendre la gestion stratégique dans une entreprise agricole suppose pour nous à la fois de comprendre une décision de pilotage stratégique en tant que telle et l'articulation de ces décisions au cours du temps ; aussi, on procédera, d'une part, à une analyse par décision et, d'autre part, à une analyse de l'articulation des décisions.

\section{Analyse par décision}

On observe tout d'abord que l'analyse de chaque décision suivant la grille proposée nous permet de comprendre tous les choix de Monsieur W., même ceux qui semblaient a priori incohérents.

Si l'on examine maintenant ce qui ressort de l'analyse globale de ces décisions appréhendées selon la grille, on constate que :

- l'agriculteur perçoit surtout des situations de problème stricto sensu, mais peu de situations d'opportunité ou de crise. Cela peut signifier que dans cette situation générale de réorganisation de son entreprise, il focalise davantage son attention sur des effets négatifs à corriger que sur des possibilités d'amélioration de l'existant; mais aussi qu'il voit cette situation générale de changement davantage comme suscitant des problèmes que comme créant des occasions d'évoluer. Monsieur W. maîtrise en tout cas relativement bien la réorganisation de son entreprise, puisque des situations de crise ne sont perçues qu'au début de la situation générale de changement, lorsqu'il n'a pas encore eu le temps de réfléchir à ses modalités d'adaptation, et que ces situations de crise ne sont que toutes relatives ;

- les cadres d'interprétation mobilisés par l'agriculteur orientent fortement la manière de formuler des problèmes et de rechercher des solutions :

- en particulier, la situation même de Monsieur W. (rupture d'association avec le frère) fait que celui-ci recherche avant tout l'autonomie dans le travail et qu'il n'envisage à aucun moment des solutions d'équipement ou de travail reposant sur des achats en commun de matériels ou un travail en entraide avec un autre agriculteur, par exemple ; 
- à l'inverse, l'agriculteur ne songe jamais à congédier son salarié permanent (ce qui est possible sur ce type d'exploitation), parce qu'il souhaite se ménager du temps libre et qu'il ne veut pas réduire son métier à celui de chauffeur ;

- enfin, le souci de simplifier le travail amène l'agriculteur à envisager les problèmes essentiellement sous l'angle de la vitesse de travail pour les matériels et sous l'angle des exigences en travail pour les productions de l'exploitation;

- sur le plan du maillage organisationnel, Monsieur W. ne prend pas en compte toutes ses cultures et toutes ses tâches, mais oriente ses raisonnements principalement par rapport à la mise en place du blé d'hiver, à celle de la betterave sucrière, à la moisson (blé et pois) et aux traitements de printemps pour résoudre les problèmes de travail; en particulier, la culture de maïs grain, la récolte des betteraves sucrières et les épandages d'engrais ne sont pas évoqués dans les problèmes de travail, ce qui peut signifier soit que l'agriculteur ne ressent pas de problème dans ces domaines, soit qu'il ne juge pas ceux-ci comme des domaines clés de réussite, ce qui semble être le cas pour le maïs grain notamment ;

- si l'agriculteur est principalement amené à résoudre des problèmes pour lesquels il ne dispose pas de solution, la recherche d'une solution satisfaisante s'effectue fortement en continuité avec des solutions connues : en particulier, Monsieur W. a nettement tendance à reproduire des solutions adoptées lorsqu'il travaillait avec son frère ou à imiter celui-ci. Des solutions radicalement nouvelles ne s'observent que dans le domaine des activités de l'entreprise (chevaux en pension et cultures fourragères, location de terres) et ne sont introduites que marginalement, dans un premier temps ;

- le poids des actions à mener au même moment joue fortement sur la formulation des problèmes (situations de crise du départ ; attention portée à une question au moment où l'action correspondante doit être menée) et sur la recherche des solutions (l'agriculteur envisage d'autant plus la «prospection» de solutions s'écartant des anciennes solutions qu'il a le temps); corrélativement, la situation financière ne joue qu'en tendance, et même si celle-ci est tendue, l'agriculteur trouve toujours des moyens pour réaliser les acquisitions de matériel dont il a besoin (leasing, achats d'occasion, rachat d'une demi-part de matériel);

- enfin, Monsieur W. doit accepter des compromis non seulement ponctuels, mais aussi sur le plan même de sa vision stratégique : la politique de simplification du travail vient notamment en partie nuancer la politique d'intensification et d'autonomie dans le travail qu'il avait au départ. 


\section{Analyse de l'articulation des décisions}

Comme on le pensait, toutes les décisions de pilotage stratégique prises n'ont pas le même statut dans le processus général d'adaptation de l'agriculteur à sa nouvelle situation. En particulier, certaines décisions sont structurantes pour les autres : ainsi, la décision de licencier l'un des deux salariés de l'exploitation conduit à modifier l'assolement et à recourir à une entreprise de travaux agricoles. D'autres décisions peuvent être vues comme des décisions ponctuelles d'ajustement : c'est le cas du choix des surfaces en pois ou en tournesol cultivées une année donnée, ou de la décision de recourir temporairement à un salarié pour certains travaux, par exemple.

Mais ces deux types de décisions sont aussi raisonnés prioritairement au sein de modules relativement déconnectés les uns des autres : les décisions de modification d'assolement, par exemple, sont traitées en relation les unes par rapport aux autres à l'intérieur d'un sous-ensemble de ce domaine de pilotage stratégique, la sole de céréales et d'oléo-protéagineux ; la surface en betteraves sucrières reste fixe et «hors raisonnement», ce qui se comprend aisément lorsqu'on sait qu'il s'agit d'une culture très rémunératrice, mais produite sous quota (donc on cherche toujours à saturer le quota permis). Les décisions concernant l'équipement sont raisonnées par bloc, à l'intérieur des cadres organisationnels des programmes d'action définis par l'agriculteur (recours à une entreprise pour la moisson, d'où acquisition d'une benne permettant de mieux équilibrer ce chantier, par exemple).

Ce raisonnement par module ne signifie pas que les décisions qui s’y rapportent soient forcément prises simultanément; au contraire, ces décisions peuvent s'étaler dans le temps, parce que tout ne peut être résolu au mieux au même moment (cas du pulvérisateur, par exemple) ou parce que de nouveaux problèmes apparaissent (cas du renouvellement de la charrue, par exemple).

Cette relative déconnexion n'exclut pas des relations entre les modules de raisonnement : ainsi, le recours à l'entreprise pour la moisson amène l'agriculteur à revendre sa moissonneuse-batteuse et à ne plus pouvoir récolter le maïs grain, donc à abandonner cette culture - par ailleurs peu rentable -, du fait du coût élevé de récolte par entreprise.

Au total se dégage l'idée d'une gestion stratégique « par morceaux ». L'agriculteur pilote le changement de façon à la fois reliée et déconnectée :

- il isole des mailles de changement - sur le plan du pilotage stratégique ou sur le plan des programmes d'action - au sein desquelles il effectue des réorganisations; celles-ci peuvent s'effectuer par rapport à des décisions structurantes, qui entraînent par exemple une suite de décisions ponctuelles d'ajustement dans cette maille (réorganisation de la sole en céréales et oléo-protéagineux après la réduction puis l'abandon du maïs 
grain) ou sous la forme d'un ensemble de décisions ponctuelles (ensemble des décisions d'équipement pour l'ensemencement des blés d'hiver);

- une modification dans une maille de changement peut par ailleurs rejaillir sur une autre, à partir d'une décision structurante (cas de la suppression d'un des deux salariés, qui entraîne des changements dans l'assolement et dans l'équipement) ou à partir d'une décision ponctuelle d'ajustement (cas du recours à une entreprise, qui amène à modifier l'assolement).

Le point important est que le « découpage » adopté par l'individu pour piloter son changement est largement fonction de ses cadres d'interprétation préexistants.

\section{Conclusion}

Nous avons montré ici qu'une approche cognitive des décisions de pilotage stratégique d'un agriculteur, s'appuyant à la fois sur une représentation des décisions dans l'entreprise agricole et sur une grille d'analyse des décisions de pilotage stratégique, permettait de comprendre celles-ci, même lorsqu'elles ne sont pas immédiates. La mise en évidence des cadres d'interprétation de l'individu et la prise en compte des traits cognitifs d'un processus de résolution de problème jouent notamment un rôle important dans cette compréhension.

S'il est bien sûr nécessaire de tester cette démarche pour d'autres agriculteurs et d'autres types d'exploitations agricoles, on peut déjà esquisser quelques pistes pour l'aide à la décision dans le domaine du pilotage stratégique et quelques voies de recherche.

\section{Implications pour l'aide à la décision en matière de gestion stratégique}

Dans la mesure où c'est l'interprétation que l'agriculteur peut avoir de sa propre situation à un moment donné qui joue sur les décisions qu'il prend, cela signifie que tout problème et toute solution sont éminemment relatifs à un individu. L'aide à la décision dans le domaine du pilotage stratégique ne doit donc pas tant se concentrer sur les résultats des décisions que sur les processus de décision qui y conduisent et doit accorder une place importante à l'aide à la formulation des problèmes.

L'intervenant extérieur joue alors d'abord un rôle d'ordre maïeutique (Berry, 1986), ce qui n'exclut pas un travail sur les comportements de gestion de l'entreprise (Attonaty et Soler, 1992): il s'agit alors d'aider l'acteur à se distancier pour prendre conscience de ses marges de manœuvre et des limites de ses constructions, sur le plan de ses cadres d'interprétation comme sur celui de ses modalités de prise de décision. 
Sur ce dernier aspect en particulier, il convient de voir que les traits de comportement d'un individu agissant avec une rationalité limitée traduisent un comportement intelligent de celui-ci pour agir dans un monde complexe et incertain. Cependant, ces mêmes traits de comportement peuvent aussi conduire à des erreurs importantes. Il s'agit donc d'aider l'acteur à prendre conscience du fait que ses interprétations sont soumises à des biais (Hogarth, 1980; Schwenk, 1984). Par exemple, il peut être utile pour un agriculteur de s'apercevoir que le principe de simplification peut l'amener à se focaliser sur un aspect particulier de sa situation, alors qu'il recherche une solution pour l'ensemble de son entreprise; que le principe de continuité peut l'amener à ne pas remettre en cause des décisions prises antérieurement, même si celles-ci ne donnent pas les résultats escomptés, à reproduire toujours la même solution pour une tâche donnée et à ne pas repérer de nouvelles solutions possibles ; que le principe de connexion étroite aux actions à mener au même moment peut l'inciter à mettre en avant l'expérience malheureuse d'une année donnée ou sa situation du moment, alors que, dans le futur, il a toutes les chances de ne pas revivre les mêmes conditions.

Dans cette optique, une grille d'analyse comme celle que nous avons proposée, accompagnée d'un guide d'entretien, peut, selon nous, fournir un support à une interaction agriculteur-conseiller:

- en ordonnant l'investigation du conseiller et en permettant que le jeu de questions-réponses ne s'effectue pas «au hasard» ou au gré de la seule expertise de celui-ci ;

- en enrichissant les tableaux de bord proposés par certains auteurs (Hémidy, Maxime et Soler, 1993), qui restent souvent trop généraux pour pouvoir réellement être utiles à l'agriculteur parce qu'ils ne tiennent pas suffisamment compte des cadres d'interprétation propres à chaque individu.

\section{Nouvelles voies de recherche}

Parmi les différentes questions qui surgissent, nous souhaiterions ici mettre l'accent sur trois d'entre elles qui nous semblent particulièrement importantes et qui renvoient respectivement :

- à la grille d'analyse construite ;

- à la représentation des décisions dans l'entreprise agricole proposée ;

- au point de vue adopté pour l'étude, laquelle porte sur la décision individuelle.

Si nous avons considéré qu'une décision de pilotage stratégique relevait d'un processus de résolution de problème, on s'aperçoit qu'aucune situation de décision ne correspond à la définition que nous avons donnée de ce type de processus dans 
l'exemple choisi : en effet, l'agriculteur formule à chaque fois des problèmes relativement bien identifiés et pour lesquels il a une idée des solutions possibles. Cela n'est pas étonnant en soi puisque l'on considère que ce type de décision obéit aussi à une logique d'adéquation ; mais on peut se demander si un individu peut réellement se trouver dans une situation de résolution de problème stricto sensu et quel sera alors son comportement. De plus, si nous avons considéré que l'agriculteur s'appuie sur des représentations préexistantes pour résoudre ses problèmes et que la recherche d'une solution s'effectue avec une complexité croissante, on ne sait pas comment s'introduisent les germes de nouveauté. Jusqu'où l'individu recherche-t-il des solutions nouvelles ou au contraire accepte-t-il des solutions qu'il « rencontre » et qui lui paraissent raisonnables? Jusqu' où le caractère de nouveauté est-il accepté par l'individu?

Par ailleurs, la notion de représentations stables doit être nuancée. En effet, pour comprendre une décision de pilotage stratégique, nous cherchons à mettre en évidence les cadres d'interprétation mis en œuvre par l'agriculteur au moment de la décision ; ceux-ci sont donc définis de façon instantanée. Or, ces cadres d'interprétation évoluent aussi, comme nous le montre aisément une étude longitudinale : les cadres organisationnels se modifient évidemment au gré des décisions d'équipement prises par exemple, mais les croyances aussi ; ainsi, dans l'exemple choisi, on s'aperçoit que Monsieur W. accepte progressivement une certaine perte d'autonomie dans son travail en faisant appel à une entreprise de travaux agricoles, alors que la recherche d'autonomie dans le travail était justement un fondement de la réorganisation de son exploitation. Une question importante est donc de mieux cerner la dialectique prises de décision / évolution des cadres d'interprétation ; une autre est de comprendre la manière dont se forgent les croyances d'un individu.

Enfin, si nous avons adopté le point de vue de l'analyse des processus de décision d'un décideur unique, l'exemple que nous avons choisi révèle l'importance du rôle que peuvent jouer d'autres acteurs dans la prise de décision d'un individu et la nécessité de mieux appréhender ce rôle. Cette question se divise en deux aspects, selon que l'on s'intéresse aux rôles d'autres acteurs qui ne cherchent pas en tant que tels à intervenir dans le processus de décision d'un individu (autres agriculteurs, par exemple), ou bien aux rôles d'acteurs amenés à intervenir dans le processus de décision (marchand de matériel, voisin avec lequel on s'associe, par exemple).

Toutes ces questions rejoignent les réflexions sur l'apprentissage organisationnel. Nous pensons notamment aux travaux d'Argyris et Schön (1978) qui distinguent un apprentissage en simple boucle, relevant d'une logique instrumentale (l'organisation prend des décisions pour améliorer une situation, mais sans modifier les représentations sous-jacentes) d'un apprentissage en double boucle, où l'organisation doit faire évoluer ses représentations pour développer de nouvelles pratiques; nous suggérons ici que ces deux types d'apprentissage ne sont pas disjoints. De 
façon générale, notre travail se situe clairement dans la perspective d'un approfondissement de la connaissance des conditions et des modalités de l'apprentissage organisationnel.

\section{Bibliographie}

Allain, S. (1997), «Approche cognitive du pilotage stratégique de l'entreprise agricole : le cas des décisions d'équipement en grande culture (fondements théoriques et étude de cas multiple) », Thèse de doctorat de l'INA-PG, Paris.

ARGYRIS, C. et D.A. SCHÖN (1978), Organizational Learning. A Theory of Action Perspective, $2^{\mathrm{e}}$ édition, Reading, Addison-Wesley, 1996.

Attonaty, J.M. et L.G. SOLER (1992), «Aide à la décision et gestion stratégique : un modèle pour l'entreprise agricole », Revue Française de Gestion, mars-avril-mai, p. $45-54$.

AVENIER, M.J. (1988), Le pilotage stratégique de l'entreprise, Paris, Presses du CNRS.

BERRY, M. (1986), «Connaissance et action. De la balistique à la maïeutique », $C R G$, Paris, École polytechnique, $14 \mathrm{p}$.

BESSON, P. et H. Bouquin (1991), «Identité et légitimité de la fonction de contrôle de gestion », Revue Française de Gestion, janvier-février, p. 60-71.

CARRIÈRE, J.-B. (1991), « La vision stratégique en contexte de PME : cadre théorique et étude empirique », Revue internationale PME, vol. 4, n ${ }^{\circ}$ 1, p. 34-56.

CERF, M. et M. SeBILlOTTE (1997), «Approche cognitive des décisions de production dans l'exploitation agricole. Confrontation aux théories de la décision», Économie Rurale, $\mathrm{n}^{\circ} 239$, mai-juin, p. 11-18.

CossetTE, P. (1996), «La vision stratégique du propriétaire-dirigeant de PME : étude de cartographie cognitive », Revue internationale PME, vol. 9, $\mathrm{n}^{\circ}$ 1, p. 123-142.

CYERT, R.M. et J.G. MARCH (1963), A Behavioral Theory of the Firm. Attempts to Develop a Theory of the Firm Which Is Based on Empirical Studies of Decision-making Within the Firm, $3^{\mathrm{e}}$ édition, Englewood Cliffs, New Jersey, Prentice-Hall Inc., 1965.

FILION, L.J. (1991), Visions et relations : clefs du succès de l'entrepreneur, Montréal, Les Éditions de l'Entrepreneur.

HÉMIDY, L., F. MAXIME et L.-G. SOLER (1993), «Instrumentation et pilotage stratégique dans l'entreprise agricole », dans L.G. Soler (dir.), Instrumentation de gestion et conduite de l'entreprise, Cahiers d'économie et de sociologie rurales, $\mathrm{n}^{\circ} 28$, Paris, INRA.

Hoc, J.-M. (1987), Psychologie cognitive de la planification, $2^{\mathrm{e}}$ édition, Grenoble, Presses universitaires de Grenoble, Sciences et Technologies de la connaissance, 1992.

HogarTh, R. (1980), Judgement and Choice. The Psychology of Decision, $2^{\mathrm{e}}$ édition, New York, John Wiley and Sons, 1987.

LAROCHE, H. et J.P. NIOCHE (1994), «L'approche cognitive de la stratégie d'entreprise », Revue Française de Gestion, ${ }^{\circ}$ 99, juin-juillet, p. 64-78. 
Le Ny, J.-F., J. Rostel, S. Moscovici, A. Reuchlin et E. Vurpillot (1992), Grand Dictionnaire de la Psychologie, Paris, Larousse.

MARCH, J.G. (1994), A Primer on Decision Making. How Decisions Happen, New York, The Free Press.

MARCH, J.G. et H.A. Simon (1958), Organizations, New York, Wiley.

Martinet, A. C. (1983), Stratégie, Paris, Vuibert Gestion.

MintzBerg, H. (1978), « Patterns in strategy formation», Management Science, vol. 24, $\mathrm{n}^{\circ} 9$, mai.

MinTZBerg, H. (1994), The Rise and Fall of Strategic Planning, New York, The Free Press, A Division of Macmillan.

Mintzberg, H., D. RAisinghani et A. ThÉORet (1976), «The structure of unstructured decision processes », Administrative Science Quarterly, juin, p. 246-275.

PetTigrew, A.M. (éd.) (1987), The Management of Strategic Change, Oxford, Basil Blackwell.

RICHARD, J.F. (1990), Les activités mentales : comprendre, raisonner, trouver des solutions, Paris, Armand Colin.

SCHWENK, C.R. (1984), «Cognitive simplification processes in strategic decision-making », Strategic Management Journal, vol. 5, p. 111-128.

Simon, H.A. (1945), Administrative Behavior. A Study of Decision-Making Processes in Administrative Organization, New York, The Free Press, A Division of Macmillan Publishing Co. Inc.

VARELA, F.J. (1988), Connaître les sciences cognitives. Tendances et Perspectives, Paris, Seuil.

WEICK, K.E. (1969), The Social Psychology of Organizing, 2e édition, Reading, New York, McGraw-Hill Inc., 1979. 